\title{
Bases juridiques pour le quotidien du médecin - un guide pratique
}

\author{
De nombreuses dispositions juridiques accompagnent le quotidien des méde- \\ cins. Il s'avère souvent difficile pour un médecin de conserver une vue d'en- \\ semble des normes juridiques applicables. Le guide «Bases juridiques pour le \\ quotidien du médecin» de l'ASSM et de la FMH lui apporte une aide précieuse \\ à cet égard.
}

\author{
Hanspeter Kuhn ${ }^{a}$, \\ Hermann Amstad ${ }^{b}$ \\ a avocat, secrétaire général adjoint \\ de la FMH \\ b Dr méd., secrétaire général \\ de l'ASSM
}

Ce guide répond de manière simple et pragmatique aux questions fréquentes et courantes. Il tient compte des textes législatifs en vigueur et des jugements rendus jusqu'en mai 2008. De par sa conception, il fait suite à la série de guides pour la pratique de la médecine qu'avait initiée l'ASSM. En ce qui concerne la FMH, ce guide succède au vadémécum épuisé depuis des années. Il est disponible dès maintenant en allemand. La version française paraîtra très probablement cette année encore.

\section{Concentration sur les aspects pratiques - version électronique régulièrement complétée}

Plutôt que sur les documents de base de la FMH autrefois contenus dans le Vademecum du médecin suisse et disponibles actuellement sur Internet tels les statuts, le Code de déontologie, etc., les 128 pages du guide se concentrent sur les questions juridiques les plus fréquentes et les plus importantes dans la pratique et au sein des hôpitaux. Les textes sont délibérément succincts et donnent une première approche du sujet. Le guide peut aussi être consulté sur le site Internet de la FMH (www.fmh.ch $\rightarrow$ Nos prestations $\rightarrow$ Droit) et il est possible de procéder à des recherches sur la base de mots-clés. Dans les mois à venir, le Service juridique de la FMH complétera cette version électronique par des indications complémentaires et des informations actuelles.

\section{Equipe d'auteurs interdisciplinaire}

Le texte de base a été rédigé par une équipe d'auteurs diversifiée, composée de collaborateurs du Service juridique de la FMH, de l'ASSM, de l'OFSP, ainsi que d'autres auteur(e)s externes. La commission de rédaction réunissant Mathis Brauchbar, Michelle Salathé, Hermann Amstad et Hanspeter Kuhn, en a assuré la congruence linguistique et didactique. Le travail du conseil scientifique, composé des professeur(e)s Christian Brückner, Olivier Guillod, Christoph A. Meier, Ulrich Meyer, Brigitte Tag et Peter Tschudi, a consisté à porter un oil critique sur la rédaction.

\section{Points essentiels}

- Le premier chapitre présente les «Fondements du droit»: la fonction du droit dans la vie quotidienne du médecin et la hiérarchie des normes, de la Constitution fédérale aux réglementations privées de l'ASSM et de la FMH.

- Le deuxième chapitre donne un aperçu systématique des «Conditions générales de l'activité médicale»: la formation prégraduée, postgraduée et continue, les devoirs professionnels et la législation sur les médicaments font partie des thèmes abordés, de même que la radiologie, les laboratoires, l'assurance-qualité, l'accréditation et la certification, sans oublier les rudiments des assurances sociales et privées.

Les chapitres suivants adoptent une approche fondée sur les problèmes:

- Le chapitre 3, «Fondements du traitement des patientes et des patients», traite du contrat de soins, de l'information du patient, de son consentement et de la tenue du dossier médical. Il explique par ailleurs les dispositions essentielles en matière de soins de la LAMal, de la LAA, de la LAM et de la LAI.

- Le chapitre 4, «Situations particulières susceptibles de se présenter lors de la prise en charge des patientes et des patients», explique les lois spéciales: des analyses génétiques à la prise en charge médicale de requérants d'asile et de patients en cours d'exécution de peine, en passant par la procréation médicalement assistée, l'interruption de grossesse non punissable et l'assistance au suicide. 
- Le cinquième chapitre se penche sur la recherche et les relations avec l'industrie.

- Le sixième chapitre traite du médecin en tant que rédacteur de rapports et «émetteur» de factures: les questions abordées vont du secret médical aux rapports effectués dans le cadre de procédures d'asile en passant par la protection des données et les rapports aux confrères, aux employeurs et aux assurances sociales et privées.

- Le septième chapitre est consacré au «Médecin en tant qu'expert» et contient les règles générales applicables à l'expertise médicale et aux particularités de l'expertise en matière d'assurances sociales.

- Le huitième chapitre traite de l'«Obligation du médecin de rendre compte»: du droit du patient de consulter et de copier son dossier médical à la responsabilité civile et pénale [du médecin].

- Le neuvième chapitre est destiné à aider le «Médecin salarié ou médecin employeur», de son engagement au sein d'un hôpital en passant par l'ouverture de son cabinet aux dispositions relatives à la protection des assistantes médicales en cas de maternité.

Une liste des auteur(e)s et des membres du conseil scientifique et un index détaillé des mots-clés concluent le guide.

Les membres de la FMH peuvent se procurer gratuitement ce guide au moyen de la carte de commande annexée ci-joint. Pour les non membres, le prix du guide s'élève à 25 francs. 